\title{
Continuous Intravascular Blood Gas Monitoring
}

\author{
Joyce A. Wahr, MD, and Kevin K. Tremper, PhD, MD
}

\begin{abstract}
$\mathbf{A}^{N}$ N AVERAGE adult at rest consumes approximately 100 million trillion molecules of oxygen per second; with virtually no capacity for oxygen storage, cessation of oxygen delivery quickly results in irreversible cellular damage. The human cardiopulmonary system functions remarkably well in healthy individuals, maintaining the mitochondrial oxygen tension (greater than $1 \mathrm{mmHg}$ ) required for aerobic metabolism. In the critically ill and in anesthetized patients, however, any acute dysfunction may result in hypoxia. The need for oxygen measurcment in blood was long apparent, but it was not until Clark developed the polarographic electrode in the 1950 s that this became routinely available in the clinical setting. ${ }^{1}$ With the addition of the carbon dioxide electrode by John Severinghaus in 1958 , the blood gas analyzer was developed. ${ }^{2}$ Today it is difficult to imagine treating patients in an acute care setting without the immediate availability of blood gas analysis. Still, there are limitations in using intermittent measurement to evaluate such a dynamic system. Ideally, blood gas data would be provided continuously so that oxygenation, ventilation, and acid-base status could be monitored and adverse changes treated before impacting the patient's overall condition. Decreasing the number of blood samples drawn would provide other benefits such as decreasing the risk of iatrogenic infection or anemia, exposure of personnel to blood-borne diseases, and the possibility of analysis error.
\end{abstract}

Continuous noninvasive monitoring of oxygenation and ventilation with pulse oximetry and capnography represents a substantial improvement in patient monitoring but does not replace arterial blood gas and $\mathrm{pH}$ analysis. For nearly two decades efforts have been made to adapt the electrochemical sensors used in in vitro blood gas measurements to continuous in vivo monitoring, but these efforts have met with limited success. ${ }^{3-5}$ Over the past 5 years, significant advances in continuous blood gas monitoring have been made using photochemical sensors, often called "optodes." 6 This article will first briefly review the electrochemical technology used in blood gas analyzers, and then describe the development and design of optically based sensors. This

From the University of Michigan, Department of Anesthesiology, Ann Arbor, $M I$.

Address reprint requests to Joyce $A$. Wahr, MD, Department of Anesthesiology, University of Michigan, UH-1G323-Box 0048, $1500 \mathrm{E}$ Medical Center Dr, Ann Arbor, MI 48109-0048.

Copyright $\circledast 1994$ by W.B. Saunders Company 1053-0770/94/0803-00I

Key words: arterial oxygenation, optodes, photochemical sensors discussion will conclude with a review of the current clinical performance data and present the different manufacturers' monitors, which are in various stages of clinical development.

\section{ELECTROCHEMICAL SENSORS}

Electrochemical devices, or electrodes, measure a small current or voltage resulting from a chemical reaction at the sensor surface. The substance being measured is not only involved in the reaction, but its concentration is the "ratelimiting" constituent of the reaction. The current or voltage change produced by the reaction is calibrated to the concentration of the substance being measured. In 1956, Leland Clark presented a small electrochemical sensor composed of a platinum cathode and silver anode ${ }^{1}$ (Fig 1). A polarizing potential is maintained between the two electrodes, which are covered with an oxygen-permeable membrane and then immersed in an electrolyte solution. Oxygen diffuses into the electrode cell and the following reaction takes place at the cathode surface:

$$
\mathrm{O}_{2}+2 \mathrm{H}_{2} \mathrm{O}+4 \mathrm{e}^{-} \rightarrow 4 \mathrm{OH}
$$

The current produced by this reaction is proportional to the oxygen partial pressure. This oxygen electrode has been used to measure oxygen tension in the gas or liquid phase ever since its initial description.

At the time Clark described his oxygen electrode, measurement of $\mathrm{pH}$ was accomplished with the " $\mathrm{pH}$-sensitive glass" electrode. When this $\mathrm{pH}$ electrode is placed in a solution, a small voltage proportional to the hydrogen ion concentration develops between the electrode and a reference ground. In 1958 John Severinghaus presented a method of mcasuring carbon dioxide partial pressure using the available $\mathrm{pH}$ glass electrode placed in a buffered electrolyte cell. ${ }^{2} \mathrm{CO}_{2}$ diffusing through a membrane into the electrolyte cell reacts with water producing carbonic acid thus changing the $\mathrm{pH}$ of the cell. This $\mathrm{pH}$ change is measured with the glass $\mathrm{pH}$ electrode, and the $\mathrm{CO}_{2}$ partial pressure is referenced to the voltage change as measured by the $\mathrm{pH}$ electrode. By 1960 , these three electrodes had been placed into one device and the blood gas analyzer was born.

Over the past 30 years, attempts have been made to miniaturize these electrodes for continuous in vivo monitoring of blood gases. Because the sensing element in the $\mathrm{pH}$ and $\mathrm{PCO}_{2}$ electrodes is glass, these devices were never viable candidates for intravascular sensors. The primary problem of electrochemical intravascular $\mathrm{PO}_{2}$ monitoring has been miniaturization of the Clark electrode. This problem has been approached in two ways. In one method 


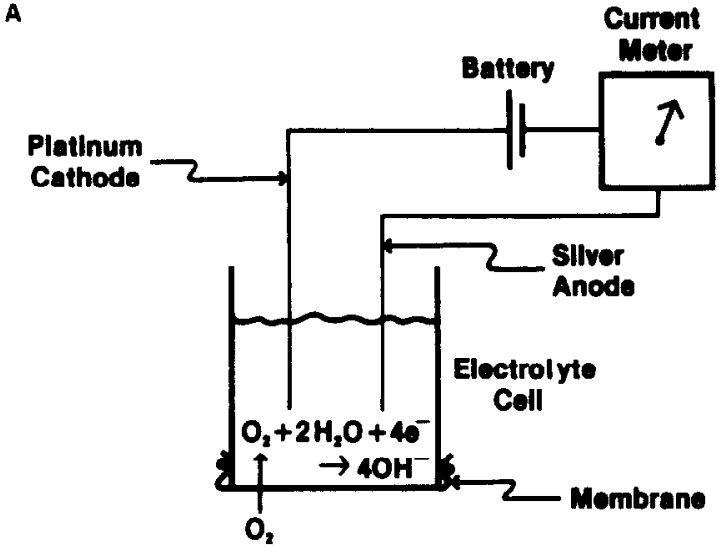

B

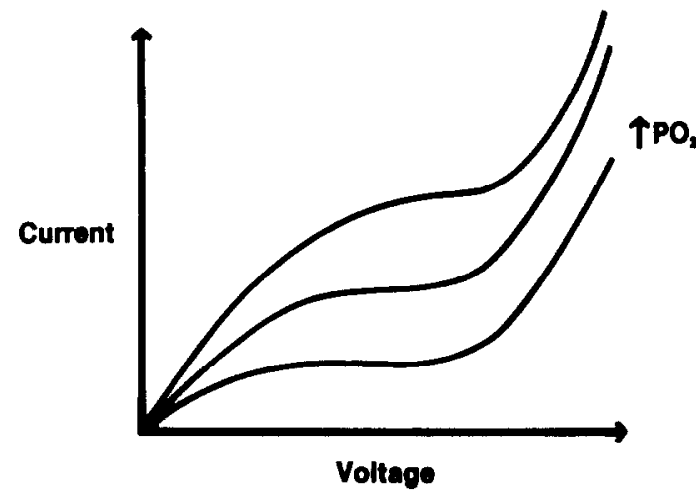

Polarogram

Fig 1. (A) Schematic of a Clark polarographic oxygen electrode. The circuit consists of a voltage source (battery) and a current meter connecting platinum and silver electrodes. The electrodes are immersed in an electrolyte cell. A membrane permeable to oxygen but not to the electrolyte covers one surface of the cell. Oxygen diffuses through the membrane and reacts with water at the platinum cathode to produce hydroxyl ions. The ammeter measures the current produced at the cathode by the electrons consumed in this reaction. (B) $A$ plot of current produced as a function of the voltage between the two eloctrodes (polarizing voltage). This plot is callod a polarogram. In the range near $660 \mathrm{mV}$ there is a plateau in the polarogram that occurs at higher currents as the $\mathrm{PO}_{2}$ in the cell is increased. Most polarographic oxygen electrodes use 600 to $800 \mathrm{mV}$ polarizing voltage to obtain a stable current at each $\mathrm{PO}_{2}$.

the platinum cathode is covered by an oxygen-permeable membrane and inserted into the blood stream whereas the anode is applied topically to the skin surface. ${ }^{5}$ In the second approach, both the anode and cathode are miniaturized to produce a small intra-arterial probe, which is surrounded by electrolyte solution and covered by a membrane. Although data have been reported from clinical studies using both approaches, the accuracy has not been promising, with problems related to technical difficulties with electrode placement, drift, thrombus formation, emboli, and interference with arterial waveforms..$^{3-5}$ Even if these problems are overcome by increased miniaturization, intra-arterial electrochemical measurement of $\mathrm{pH}$ and $\mathrm{PCO}_{2}$ is not feasible. In spite of the difficulty experienced translating these electrochemical devices to intravascular monitors, they remain the mainstay of blood gas analyzer technology today.

\section{PHOTOCHEMICAL SENSORS: OPTODES}

Just as electrodes quantify the concentration of the substance being measured on the basis of electrochemical (voltage or current) changes, optodes quantify concentrations on the basis of photochemical reactions, which produce changes in light absorption, light reflection, or light emission (luminescence or fluorescence). The two basic components of a photochemical sensor are the optical fibers that transmit light with high efficiency and the specific photochemical dyes. This section will first discuss the principles of fiberoptic light transmission and photochemical dyes, and then describe how the two are configured to produce a sensor.

Optical fibers work on the principle of total internal reflection (Fig 2). These fibers consist of a central core of glass or plastic that is clad with another material whose refractive index is much higher than that of the core material. ${ }^{6}$ The refractive index of a substance, $\mu$ (which is dimensionless) is the speed of light in a vacuum (C) divided by the speed of light in that substance (V):

$$
\mu=\frac{C}{V}
$$

When the $\mu$ of the cladding material is much greater than the $\mu$ of the core material, light striking the interface between the two is nearly completely internally reflected, resulting in efficient transmission of light down the fiber. Over the past two decades optical fibers have become ubiquitous in almost every industry.

The second critical material for development of optodes is the photochemical dye. When photons strike a substance,
Fig 2. Transmission of light by fiberoptic strand. Nearly complete internal reflection can be achieved if the cladding material has a refractive index $\left(\mu_{2}\right)$ that is much greater than that of the core material $\left(\mu_{1}\right)$. These fibers can be produced with very small diameters $(<1 \mu \mathrm{m})$ and yet trans. mit light with very high efficiency.

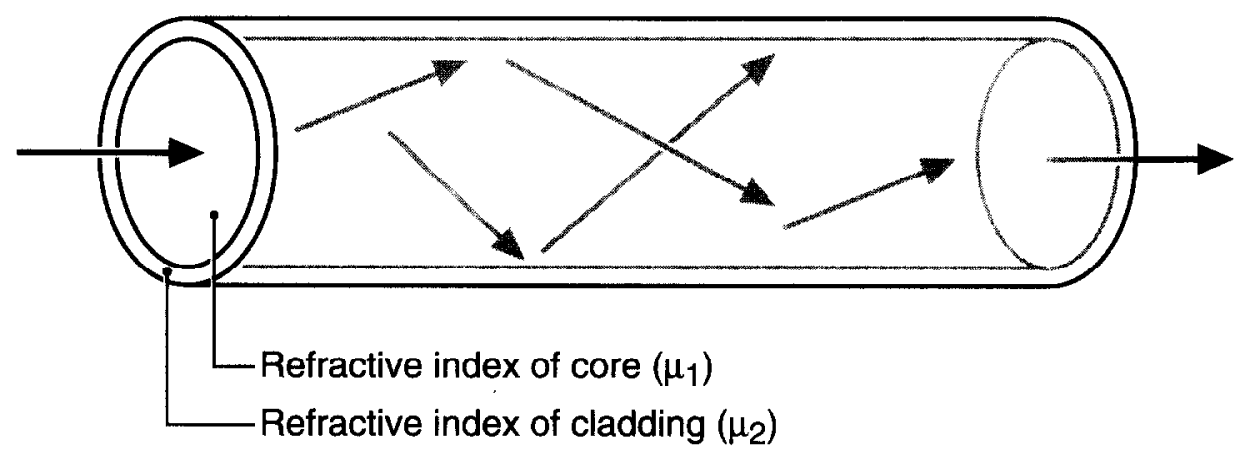




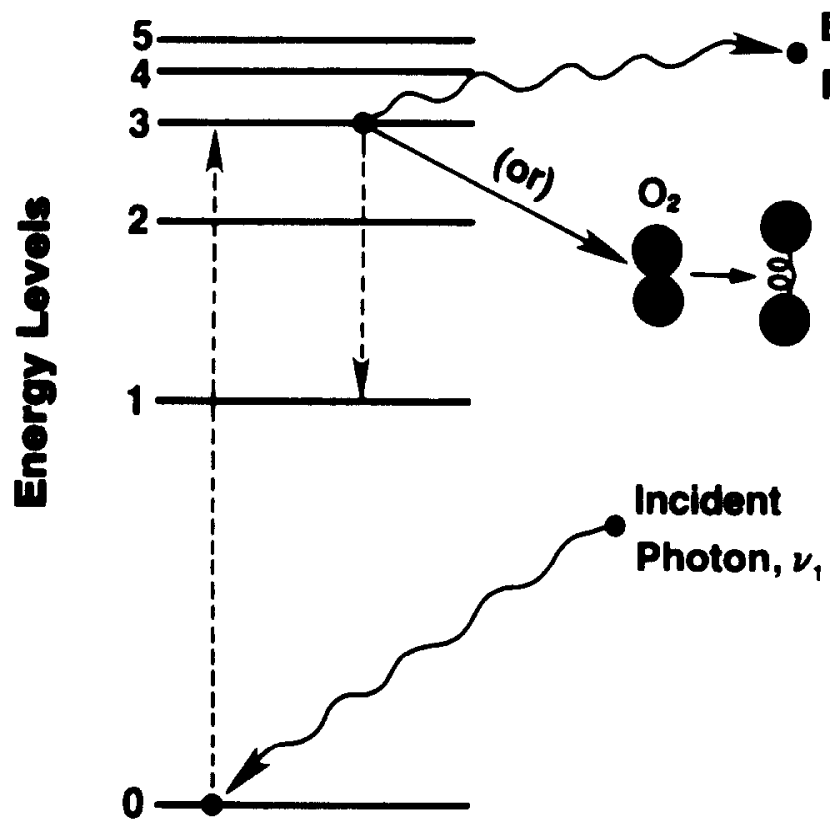

Emitted Photon, $\nu_{2}$ the light can be transmitted, reflected, absorbed, or absorbed and re-emitted. ${ }^{7,8}$ Substances that re-emit absorbed light are called fluorescent, phosphorescent, or luminescent, depending on the photochemical properties of the dye involved. ${ }^{8}$ When the energy of a photon is absorbed by a luminescent dye, electrons in the dye are elevated to a higher energy state. After a small time lag, that photon of light is re-emitted as the electron drops back to its ground state (Fig 3). In certain dyes this re-emitted energy can be absorbed by oxygen molecules, "quenching" the intensity of the luminescent signal.,10 The intensity of the luminescent signal reliably decreases as the concentration of oxygen increases. The theoretical relationship between luminescent intensity and oxygen concentration is given by the Stern Volmer equation:

$$
\frac{\mathrm{L}_{0}}{\mathrm{~L}}=\mathrm{K}\left(\mathrm{PO}_{2}\right)+1
$$

where $\mathrm{L}_{0}$ is the intensity of the luminescent signal in the absence of oxygen, $L$ is the intensity of the signal in the presence of oxygen, and $\mathrm{K}$ is the quenching constant. Equation 3 can be rearranged to the following:

$$
\mathrm{PO}_{2}=\frac{\left[\left(\frac{\mathrm{L}_{0}}{\mathrm{~L}}\right)-1\right]}{\mathrm{K}}
$$

The degree of change in the intensity of luminesced light is much greater at low concentrations of oxygen than at higher ones, indicating that the luminescent quenching techniques would be more accurate at low oxygen tensions. The luminescence quenching method for measurement of oxygen tension has been known for years but its application with optical fibers to produce oxygen sensors has come about only in the past decade..$^{6,9-12}$ An oxygen probe can be constructed by placing an oxygen-sensitive dye (with a
Fig 3. The fluorescence quenching phenomenon. An electron of the fluorescent dye is excited to a higher energy level by an incident photon $\left(v_{1}\right)$. This excited electron can return to a lower energy level by either emitting a photon $\left(v_{2}\right)$ or interacting with an oxygen molecule and raising the latter to a higher vibrational energy level. (Reprinted with permission. $\left.{ }^{8}\right)$ known quenching constant, K) at the tip of a fiberoptic strand. A xenon lamp emits light of a known intensity down the fiberoptic strand, and the dye re-emits an intensity of light back up the strand proportional to the oxygen tension surrounding the dye.

$\mathrm{PO}_{2}$ optodes were initially based upon quenching of luminescence intensity as described above. A second means of determining partial pressure of oxygen with optodes is based on the known time lag from excitation to re-emission of the photon, known as the "luminescence lifetime."12 This "lifetime" is reliably altered in the presence of oxygen, and $\mathrm{PO}_{2}$ therefore can be determined by pulsing incident light and measuring the shift in luminescence lifetime. The equation describing the relationship between $\mathrm{PO}_{2}$ and the lifetime shift is:

$$
\mathrm{PO}_{2}=\frac{\left[\left(\frac{\mathrm{t}_{0}}{\mathrm{t}}\right)-1\right]}{\mathrm{K}}
$$

where $t_{0}$ is the luminescence lifetime in the absence of oxygen, $t$ is the measured luminescence lifetime in the presence of oxygen, and $\mathrm{k}$ is a luminescence time constant. The theoretical advantage of using a "time shift" method to measure $\mathrm{PO}_{2}$ versus intensity quenching is that intensities of light can be distorted not only by oxygen quenching, but also by bending of the optical fiber, dye photobleaching and misalignment of optical connectors; time shift measurements are not affected by these practical considerations. ${ }^{12}$ Thus, when luminescence intensity is used, a second dye is required whose luminescence is unaffected by the presence of oxygen, thus providing a reference intensity to account for the losses of light intensity due to fiberoptic transmission characteristics.

Photochemical sensors that measure $\mathrm{pH}$ use either fluorescent or absorbance technology. ${ }^{13-15}$ Fluorescent dyes 
A

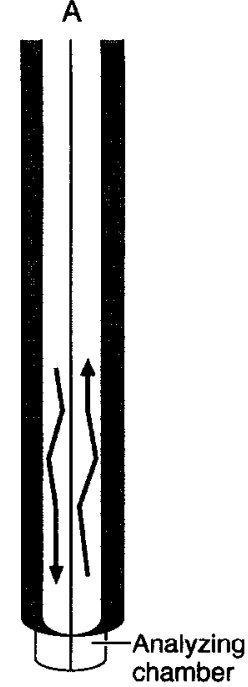

B

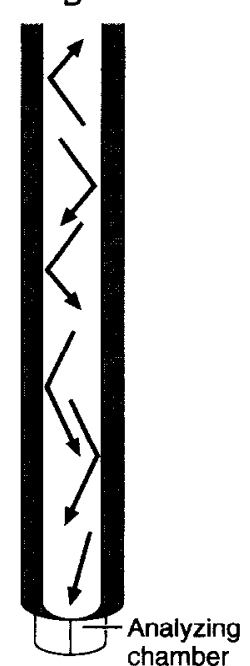

C

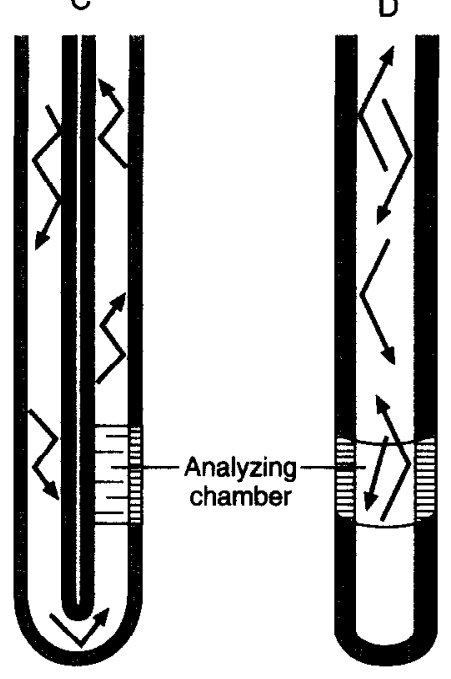

Fig 4. Schematic diegram of various probe geometries, inctuding monofiber, dual fiber, continuous tranemiesion (bent fiber), and probe using circumferential incorporation of dye matrix into covering of optic fiber. (A) dual fiber; (B) monofiber; (C) transmission; (D) monofiber with circumferential analyzing chamber. suitable for use in $\mathrm{pH}$ sensors are those that exist in both acidic and basic forms, and the proportion of the dye in each form at any time corresponds to the hydrogen ion concentration. The acidic form of the dye has a maximum cxcitation frequency differcnt from that for the basic form of the dye. The fluorescence intensity observed at each excitation frequency therefore defines the portion of dye existing in the basic versus acidic form, and $\mathrm{pH}$ is based on the ratio of the fluorescent intensity returned from each of the two exciting frequencies. Using this ratio method eliminates the problems of light intensity loss due to mechanical problems. Light absorbance can also be used to determine $\mathrm{pH}$. An indicator such as phenol red absorbs a variable portion of green light (wavelength $558 \mathrm{~nm}$ ) depending on the hydrogen ion concentration. Red light at a wavelength $600 \mathrm{~nm}$ is not absorbed at all. The device alternately flashes red and green light, and the proportion of green light absorbed relative to the reference red light is used to calculate $\mathrm{pH}$.

The carbon dioxide photochemical sensor is based on the same secondary sensing principle described by Severinghaus ${ }^{15}$ for the electrochemical $\mathrm{PCO}_{2}$ sensor. A pH-sensitive optode is bathed in a buffering solution and coated with a hydrophobic silicone layer permeable to $\mathrm{CO}_{2}$. The $\mathrm{CO}_{2}$ rapidly equilibrates across the silicone matrix, is buffered, and the resulting $\mathrm{pH}$ change, measured by either fluorescence or absorbance technology, is calibrated to $\mathrm{PCO}_{2}$.

Because of the inherent characteristics of fiberoptics and photochemical dyes, fluorescent optodes offer a number of advantages over other possible sensor designs. They are uniquely suited for miniaturization because of the availability of very small diameter yet reliable optical fibers (as small as 50 to $100 \mu \mathrm{m}$ ). The dyes used to measure gas tensions are stable over time, are biocompatible (nontoxic to the patient, and not affected by the patient environment), have a fast response time to changes in concentration of the measured substance ( 60 to $90 \mathrm{sec}$ ), and are not consumed by the measurement process. The probe assembly is relatively simple and fairly immune to interference from the environment. Perhaps most importantly, optodes do not expose the patient to electrical risk and are immune to electrical noise. Despite the inherent simplicity of optodes, the geometry of the optical fibers and the characteristics of the photochemical dye substances have posed substantial technical problems that have been addressed in a variety of ways by manufacturers of these devices.

\section{GENERAL SYSTEM DESIGN CONSIDERATIONS}

\section{Sensor Probe Design}

Both the delivery of the excitation light and the recovery of the emission light must occur with high energy efficiency to maximize accuracy. The energy efficiency is altered by fiberoptic geometry and by both the number of optical connections and the length of the optical cable through which the beam is carried. Various fibcroptic light transmission geometries are schematically illustrated in Fig 4 . Both dual and monofiber systems have the indicator dye located at the tip of the probe, a geometry with inherent loss of signal energy. Dual fibers transmit and receive light simultaneously, whereas monofiber systems alternatively send and receive. A transmission geometry using a $180^{\circ}$ bend in the fiber with the analyzing chamber at the side of the probe improves emission (returning) signal strength. The final design uses indicator impregnated circumferentially in the cladding of the fiber. This is also said to improve the strength of the returning signal. With any one of the above geometries, the strength of the excitation light signal is high but the energy of the emission light is considerably diminishcd via the losses in the analyzing chamber (either quenched as in the oxygen probe or absorbed as in $\mathrm{pH}$ and carbon dioxide probes) and through the tip of the fiber. Therefore, the length of the fiber carrying light to the patient has less impact than the length of the fiber that carries the returning light signal to the detector. Most monitoring systems consist of four components as shown in Fig 5 and as described below: the analyzer, the interface module, the display module, and the sensor probe. The interface module contains the optical multiplexer and the 
Display Module

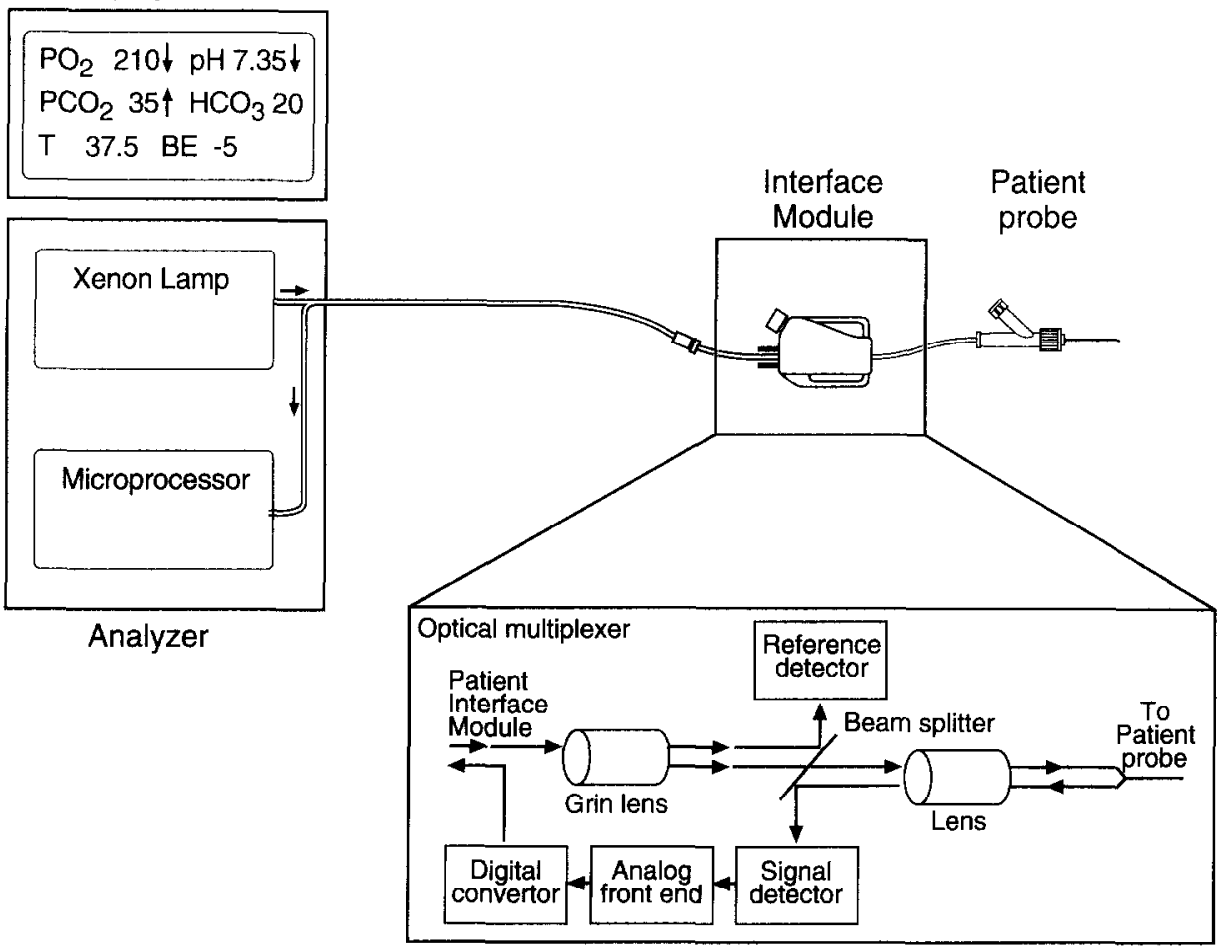

Fig 5. Schematic of a typical continuous intra-arterial blood gas monitor, consisting of the display module, the analyzer, the interface module, and the disposable patient probe (IV).

emission detector, and is located as close to the patient probe as technically feasible.

\section{Anatyzer}

The analyzer contains the light source and the microprocessor responsible for system function control, data processing, and analysis of results. Light of the appropriate wavelength is sent from the high-output light source through a lens to focus it into the interface fiberoptic cable and thus to the interface module.

\section{Interface Module}

The interface module, although complex, is still small enough to be located in close proximity to the patient. It consists of the optical multiplexer, the analog front end, and a digital converter. The optical multiplexer receives the excitation energy sent from the light suurce and passes the light through a lens for collimation. The collimated beam then passes through a beam splitter so that a portion of the light is sent to a reference detector for monitoring lamp output, while the remainder of the beam is focused into the optical fiber of the probe and sent to the analyzing chamber. The light emitted from the photochemical dye is returncd along the same fiber and is focuscd by the bcam splitter onto a broad band width detector (photo diode) for conversion from an optical to an electrical signal. This analog electrical signal is then amplified and filtered until it is acceptable for digital conversion and the digital signal is transmitted to the microprocessor.

\section{Display Module}

The digital signal received in the microprocessor is referenced both to the input lamp intensity and the refer- ence light intensity before conversion via algorithm to a recognizable blood gas value for display on the monitor. When a change in blood gas tension is detected, most systems will display the appropriate up or down arrow until equilibrium is reached (generally within 60 to 90 seconds)

\section{Sensor Probe}

The patient sensor probe, as shown in Fig 6, consists of three individual fiberoptic strands, each with its specific dye covering at the tip. In addition to the fiber bundles required for $\mathrm{PO}_{2}, \mathrm{PCO}_{2}$ and $\mathrm{pH}$ determination, blood temperature must also be continuously monitored as gas solubilities and $\mathrm{pH}$ change with temperature. A thermocouple is therefore imbedded in the fiberoptic sensor to provide a continuous measurement of blood temperature at the analyzing chamber.

Each patient probe is supplied with a calibration device both to ensure accurate calibration prior to insertion and to prevent dehydration of the probe during shipping and storage. Currently, a two-point tonometer gas calibration is required for most systems and is accomplished by bubbling two known concentrations of gas in sequence through the probe while in the calibrating device. This in vitro calibration system is very similar to those used for calibration of bench blood gas analyzers. These systems may also be calibrated as needed in vivo to laboratory arterial blood gas determinations.

\section{EXPERIMENTAL AND CLINICAL STUDIES}

As shown in Table 1, optimal probe design would include the following properties: quadruple function $\left(\mathrm{PO}_{2}, \mathrm{PCO}_{2}\right.$, $\mathrm{pH}$ and temperature), diameter small enough to permit 


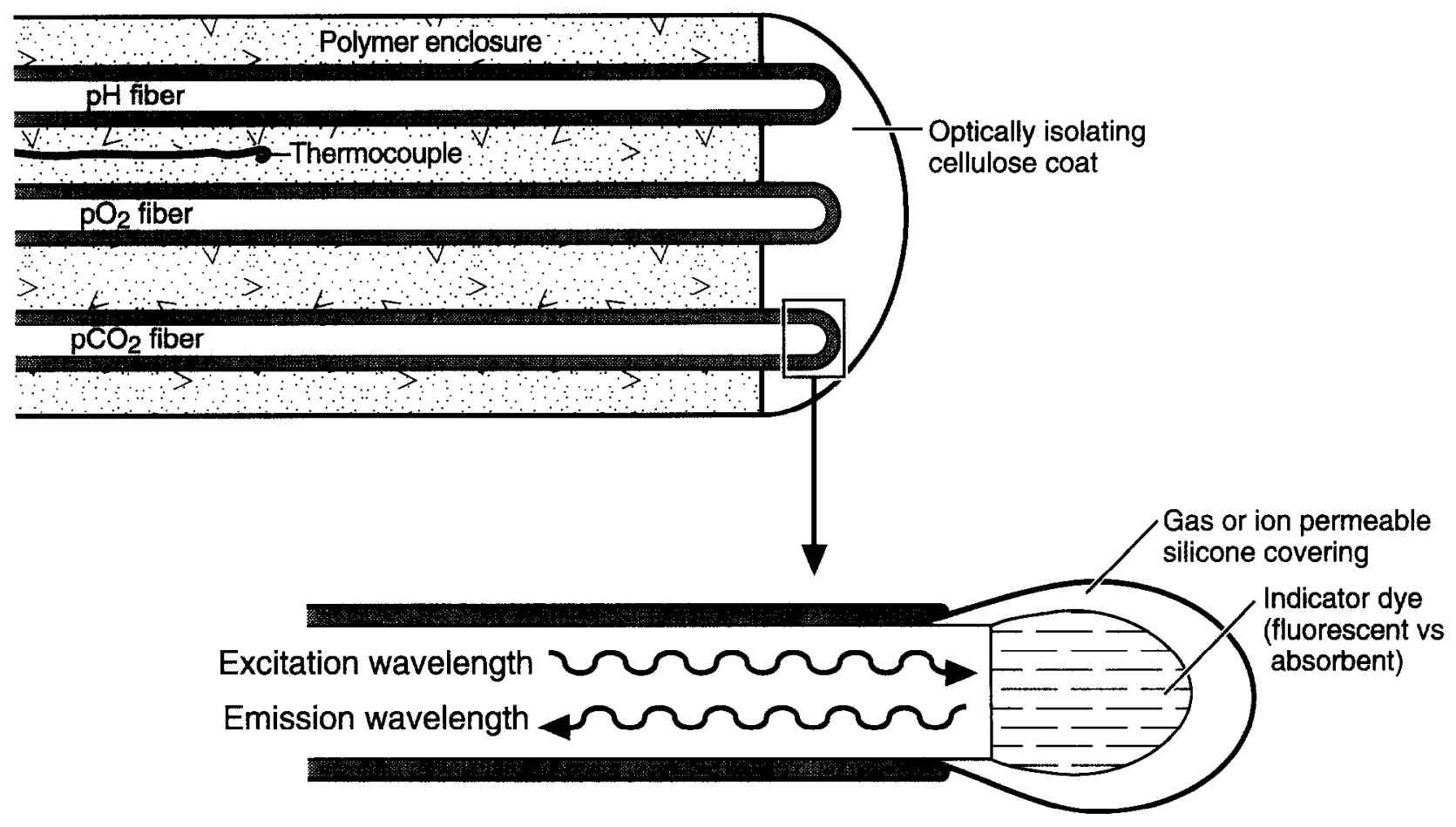

Fig 6. Schematic of a four component sensor probe for determination of $\mathrm{PO}_{2}, \mathrm{PCO}_{2}, \mathrm{pH}$, and temperature.

insertion through a 20 -gauge radial artery catheter without altering transduced pressure, nonthrombogenic and nontoxic (heparin bonded), unaffected by blood or drugs, sensitive, specific, accurate without drift, rapid response, inexpensive, and easy to use. Although all of these goals have not been accomplished to date, substantial progress has occurred over the past few years. The following section will review the accuracy data, which have been presented in the scientific literature using various optode probes. Before the specific data are presented, the most common statistical methods used to assess accuracy will be briefly reviewed, because many of the articles use different methods making it difficult to compare data from different studies.

Evaluation of the accuracy of a new measurement device usually involves determining values with the new instru-

Table 1. Desirable Characteristics of a Continuous Intra-Arterial Blood Gas Monitor

1. Be highly accurate with good precision and reliability

2. Have little or no drift over 48 to 72 hours

3. Have a rapid response time $(20$ to $60 \mathrm{sec})$

4. Be compatible with existing arterial catheter systems (20 gauge) without interfering with transduced pressures or withdrawal of blood samples

5. Be biocompatible and nonthrombogenic

6. Be unaffected by conditions of high or low cardiac output, or shock states

7. Be easy to use and maintain

8. Be cost effective, either reducing laboratory determination or reducing patient care costs

9. Have a small footprint, given the space constraints of most ICU or OR conditions ment and comparing them with ones derived from the existing technology. In this case the bench blood gas machine is considered to be the gold standard by which all blood gas monitors will be measured. Although the blood gas machine is a very reliable device, any device will have a certain degree of acceptable error. In the case of blood gas machines, electrode precision using standard electrolyte solutions are as follows: $\mathrm{pH} \pm 0.01$ unit, $\mathrm{PCO}_{2} \pm 2 \%$ (approximately $\pm 1 \mathrm{mmHg}$ at $40 \mathrm{mmHg}$ ) and $\mathrm{PO}_{2} \pm 3 \%$ (approximately $2.5 \mathrm{mmHg}$ at $80 \mathrm{mmHg}$; over $150 \mathrm{mmHg}$, the precision is $\pm 10 \%)$. ${ }^{16}$ Variability using actual blood samples may be greater, especially considering preanalytic error such as air in the syringe, inappropriate volume of heparin for the volume of blood, and time delay from sampling to measurement. Thorson et $\mathrm{al}^{17}$ drew an arterial sample each 10 minutes for 1 hour in 29 stable intensive care unit (ICU) patients, then analyzed each sample two times on the same blood gas analyzer. ${ }^{17}$ The range separating the highest and lowest $\mathrm{PO}_{2}$ in any one patient over the hour varied from 1 to $45 \mathrm{mmHg}$, and $\mathrm{PCO}_{2}$ varied from 1 to $8 \mathrm{mmHg}$. The average percent change in $\mathrm{PO}_{2}$ between sequential intrapatient samples was $5.3 \pm 2.8 \%$. Despite the known variability in gas machine values, the accuracy of intravascular blood gas optodes has been and will continue to be assessed by how well they agree with bench arterial blood gas analysis.

The classic statistical method for comparison of values of two continuous variables from two different measurement devices is linear regression. The linear regression correlation coefficient asscsscs how well values measured with the new device follow changes in values determined by the standard device. An example of linear regression data 

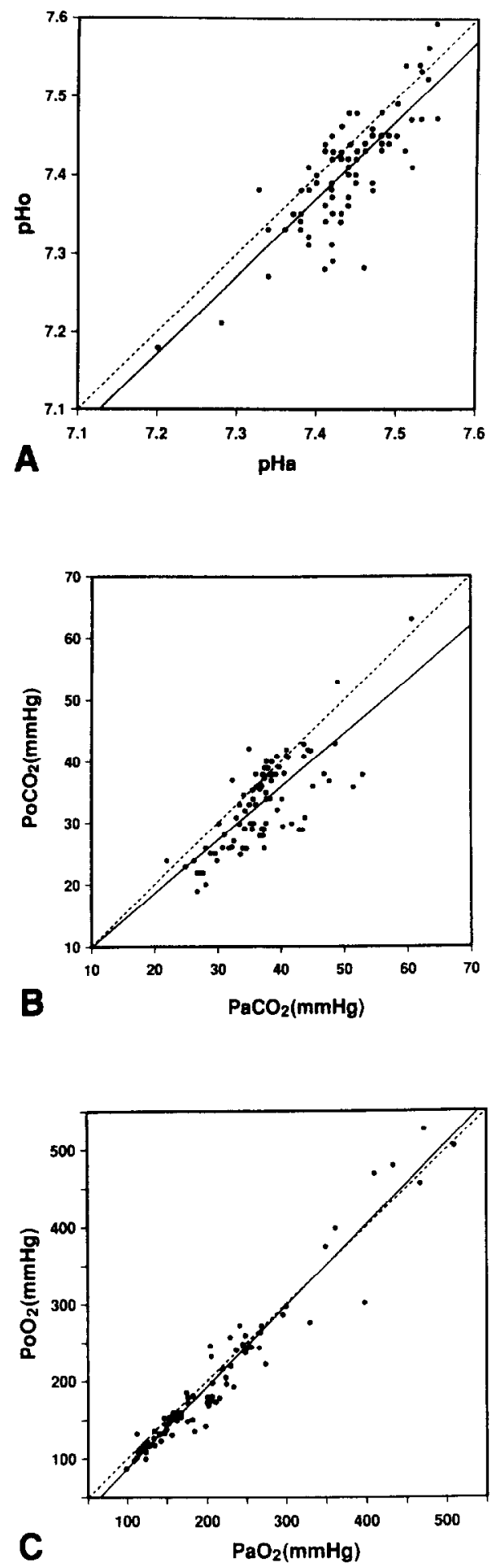

Fig 7. Linear regression plots showing optode values $\left(\mathrm{PoO}_{2}\right.$ $\left.\mathrm{PoCO}_{2}, \mathrm{pHo}\right)$ and simultaneous in vitro analyzer values $\left(\mathrm{PaO}_{2}, \mathrm{PaCO}_{2}\right.$, pHa); 14 patients, $\mathbf{N}=87$. Line of identity, --.-; linear regression, - . (Reprinted with permission. International Anesthesia Research Society.21)

taken from a study by Barker et $\mathrm{al}^{21}$ is shown in Fig 7. A correlation coefficient value close to one (for example, $r=0.98$ ) demonstrates a very high degree of positive association, ie, when one increases, so does the other.
Correlation coefficients, however, do not indicate huw much one will rise when the other rises or how closely the numerical values agree. Another weakness of the linear regression method for comparison of accuracy of two methods of measure is that the $r$ value is significantly affected by the range over which the data are collected. For example, if 10 blood gas samples show $\mathbf{P C O}_{2}$ values between 40 and $42 \mathrm{mmHg}$ and the optode readings are also between 40 and $42 \mathrm{mmHg}$, it is very difficult to determine the slope of the line connecting these data. If, however, arterial blood gas samples are collected where the $\mathrm{PO}_{2}$ values range from 60 to $400 \mathrm{mmHg}$, it will be easy to demonstrate a high degree of association (an $r$ value close to 1) with an optode device that works to any degree, even with great variability between measured values.

When trying to determine if a new method of measure is of sufficient accuracy to replace an older method of measure, it is useful to know how well the two measurement values agree over the range of clinical interest. Altman and Bland $^{18}$ have suggested a simple method of assessing this type of agreement using bias and precision. Bias, the mean difference between the paired values, represents the systematic error between two measurement methods, eg, does the optode consistently overestimate or underestimate the blood gas machine? This systematic error can be corrected by recalibrating the device. Precision is the standard deviation of the differences, and provides a measure of the random error inherent in the device. Random error cannot be correcled. A trusted device must have a very small precision. Altman and Bland also suggest plotting the data on a bias plot: the difference of the two values on the vertical axis versus the mean of the two values on the horizontal axis, with a horizontal line at the zero difference point representing the ideal. The bias plots shown in Fig 8 are from the same study as the linear regression graphs shown in Fig 7 , illustrating the information gained by the different statistical methods. Finally, if the bias changes with time, the instrument is said to drift. Significant drift would require recalibration at frequent intervals, somewhat defeating the purpose of having continuous data.

\section{Clinical Studies}

In spite of the enthusiasm over intravascular blood gas monitoring with optodes, there are only a few clinical studies to date that assess accuracy, a few case reports that may demonstrate utility, and no data that address cost/ benefit issues. The first clinical study on a triple-function optode, conducted in both dogs and humans, was presented by Shapiro et al in $1989 . .^{19}$ The probe, a $0.62-\mathrm{mm}$ diameter optode (CDI-3M Healthcare, Tustin, CA), was inserted through a 20-gauge arterial catheter. This first study used optodes calibrated in vivo to a bench blood gas analyzer and 79 blood samples collected from 12 critically ill patients over 3 to 26 hours. The bias and precision data were $\mathrm{pH}$ $0.002 \pm 0.02, \mathrm{PaCO}_{2} 0.44 \pm 2.97 \mathrm{mmHg}$, and $\mathrm{PaO}_{2} 1.22 \pm$ $9.34 \mathrm{mmHg}$ (Table 2). The excellent bias data arise from calibration of each probe in vivo with a bench blood gas analyzer first, and elimination of 13 probes because they did not calibrate. Five of the 12 probes that did calibrate failed 

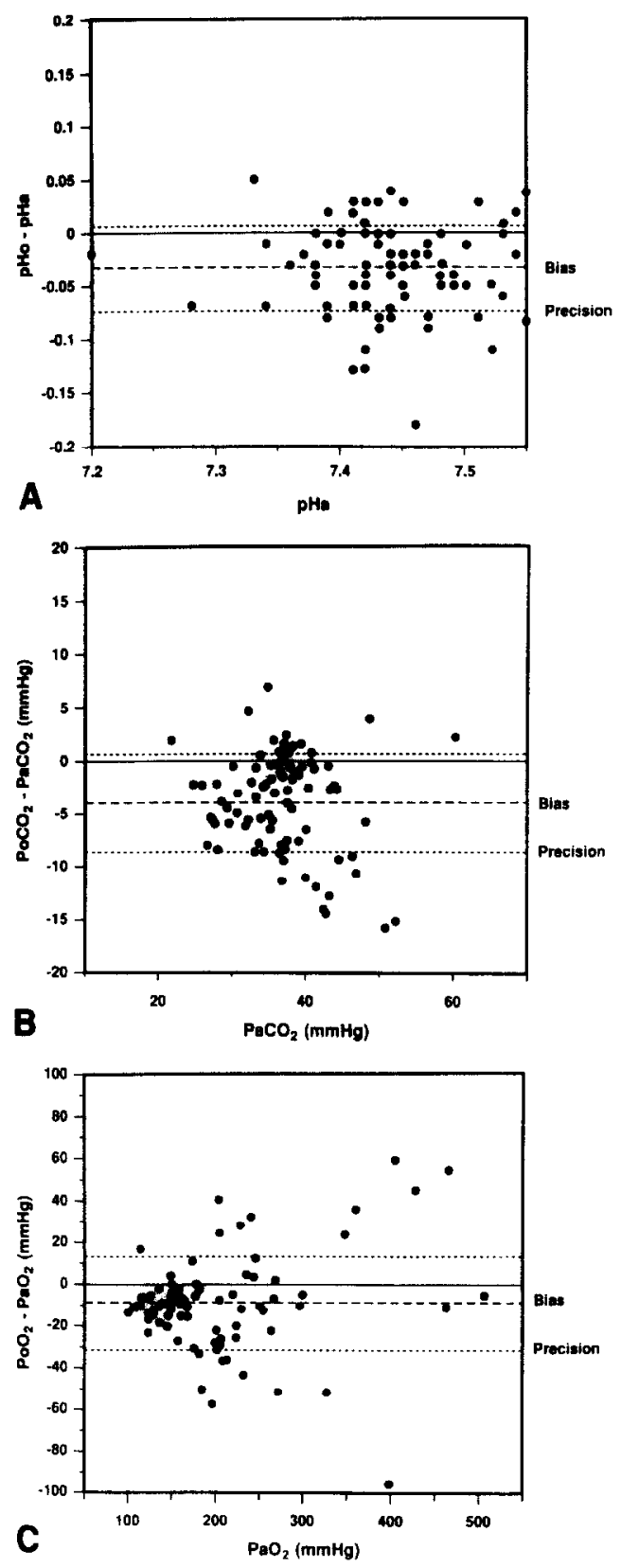

Fig 8. Bias plots showing values of error $\left(\mathrm{PoO}_{2}-\mathrm{PaO}_{2}\right.$ $\mathrm{PoCO}_{2}-\mathrm{PaCO}_{2}, \mathrm{pHo}-\mathrm{pHa}$ ) plotted versus in vitro analyzer values $\left(\mathrm{PaO}_{2}, \mathrm{PaCO}_{2}, \mathrm{pHa}\right) ; 14$ patients, $\mathrm{N}=87$. Bias or mean error, -..--; \pm precision, .... (Reprinted with permission. International Anesthesis Research Society. ${ }^{21}$

during the study and were dropped from analysis. The authors concluded that further study was warranted. ${ }^{19}$

Early clinical studies suffered from acute decreases in the oxygen optode measurement unrelated to arterial $\mathrm{PO}_{2}$ changes (Figs 9, 10). Mahutte et al $^{20}$ placed oxygen optode probes in 18 volunteers. They found two causes for these rapid and unpredictable decreases in $\mathrm{PO}_{2}$ measurement. Clot formation at the tip of the sensor caused a "down-updown" pattern with decreased $\mathrm{PO}_{2}$, increased $\mathrm{PCO}_{2}$, and decreased $\mathrm{pH}$ (Fig 9). Acute decreases in oxygen measurement could be produced when the probe was adjacent to the arterial vessel wall (Fig 10). Slight retraction of the probe returned the $\mathrm{PO}_{2}$ value to normal. Thus, the distance the probe protruded beyond the cannula was critical: overextension caused a wall effect (unpredictable decreases in oxygen values), whereas retraction too far within the cannula produced values contaminated by the flush solution. Investigation of this "wall effect" proceeded with a special probe with three oxygen sensors $120^{\circ}$ apart on the sides of the sensor tip. Rotation of the probe produced the $\mathrm{PO}_{2}$ "down" pattern as each sensor found the vessel wall, as shown in Fig 10. Table 2 contains the bias and precision data: $\mathrm{pH} 0.003 \pm 0.02, \mathrm{PCO}_{2} 0.77 \pm 2.44 \mathrm{mmHg}, \mathrm{PO}_{2} 2.9 \pm$ $25.4 \mathrm{mmHg}$. These optodes were calibrated prior to insertion into the patient by the tonometer system provided.

This study by Mahutte et al describes a crucial clinical application flaw in the probes tested at that time: acute, unpredictable, highly significant decreases in $\mathrm{PO}_{2}$ could be produced solely by position changes of the probe. These decreases were unrelated to any actual change in the patient's arterial oxygenation. The authors postulated means of correcting this problem of "wall effect" using various probe geometries, but little data exist in the literature demonstrating that altering the probe geometry does, in fact, obviate this problem.

Barker and Hyatt conducted a third clinical study intraoperatively using the same CDI-3M optode. ${ }^{21}$ That optode required an 18 -gauge radial artery catheter. Eighty-seven arterial blood samples were compared to optode values; no acute decreases in $\mathrm{PO}_{2}$ occurred. Bias and precision data revealed $\mathrm{pH}-0.032 \pm 0.042, \mathrm{PaCO}_{2}-3.8 \pm 4.7 \mathrm{mmHg}$, and $\mathrm{PaO}_{2}-9 \pm 23.3 \mathrm{mmHg}$ (Table 2). Note the negative bias values for each variable. Although these data demonstrate improved accuracy, the need for an 18-gauge catheter reduced the clinical desirability of this CDI-3M probe. Recently, CDI-3M presented an ex vivo, on demand, arterial blood gas monitoring system. That company continues to develop its intra-arterial probe, which may or may not resemble the probe used in the Barker and Hyatt study.

The largest human study to date on an optode blood gas system is the multicenter trial of the ex vivo CDI 2000 Blood Gas Monitoring System (CDI-3M Healthcare, Tustin, CA).22 This system has the sensing chamber located outside of the body. When analysis is desired, a technician withdraws an arterial sample into the sensing chamber; results are displayed after approximately 2 minutes. This design mitigates thrombogenicity and arterial wall interference, but represents a measurement rather than a monitoring technique. Nonetheless, response time is fast enough to permit very frequent (many times per hour) sampling while preserving test reliability and protecting both patients and personnel from risk of blood-borne infections. The results from this study included 1,341 samples drawn from 117 patients over 80 hours. The bias and precision values shown in Table 2 demonstrate reasonable accuracy.

Zimmermann and Gellinger ${ }^{23}$ presented data on a triple optode probe produced by Optex Corporation (The Woodlands, TX). Onc hundred four blood samples from five critically ill patients monitored over 68 hours were compared to two different bench top blood gas analyzers. The bias and precision values were $-0.021 \pm 0.037$ for $\mathrm{pH}, 1.74$ 
Table 2. Summary of Human Clinical Studies

\begin{tabular}{|c|c|c|c|c|c|c|c|c|c|c|c|c|}
\hline \multirow[b]{2}{*}{ Author } & \multirow[b]{2}{*}{ Probe } & \multicolumn{3}{|c|}{$\mathrm{PO}_{2}$} & \multicolumn{3}{|c|}{$\mathrm{PCO}_{2}$} & \multicolumn{3}{|l|}{$\mathrm{pH}$} & \multirow[b]{2}{*}{ Subject } & \multirow[b]{2}{*}{ Calibration } \\
\hline & & $B \pm P$ & I & N & $B \pm P$ & r & $N$ & $B \pm P$ & $r$ & N & & \\
\hline \multirow[t]{2}{*}{ Shapiro ${ }^{19}$ (1989) } & CDI & $-17 \pm 46$ & 0.96 & 256 & $1 \pm 4$ & 0.95 & 359 & $-0.02 \pm 0.03$ & 0.97 & 420 & Dog & In vitro \\
\hline & & $-1 \pm 9$ & 0.99 & 79 & $0.44 \pm 3$ & 0.96 & 79 & $0.002 \pm 0.02$ & 0.97 & 79 & Humant & In vivo \\
\hline Mahutte $20(1990)$ & $\mathrm{CDI}$ & $-3 \pm 25$ & 0.99 & 48 & $0.8 \pm 2.4$ & 0.98 & 48 & $0.003 \pm 0.2$ & 0.98 & 48 & Human & In vivo \\
\hline Larson $^{27}(1990)$ & PB & $3.0 \% \pm 3 \%$ * & 0.99 & 76 & $5.1 \% \pm 5.1 \% *$ & 0.92 & 93 & & & & Human & Unknown \\
\hline Barker $^{21}(1991)$ & $\mathrm{CDI}$ & $-9 \pm 23$ & & 87 & $-3.8 \pm 4.6$ & 0.87 & & $-0.03 \pm 0.04$ & 0.87 & & Human & In vitro \\
\hline Zimmermann ${ }^{24}(1992)$ & $\mathrm{CP}$ & $-2,1 \pm 13$ & & 301 & $1.5 \pm 5.5$ & & 301 & $-0.02 \pm 0.04$ & & 301 & Human & In vitro \\
\hline Shapiro 22 (1993) & $\mathrm{CDI}$ & $-2.2 \pm 8.7$ & 0.94 & 1,341 & $-0.8 \pm 2.2$ & 0.92 & 1,341 & $-0.004 \pm 0.027$ & 0.85 & 1,341 & Human & \\
\hline Zimmermann ${ }^{23}$ (1993) & $\mathrm{CP}$ & $-5.89 \pm 13.19$ & & 104 & $1.74 \pm 6.06$ & & 104 & $-0.021 \pm 0.037$ & & 104 & Human & \\
\hline
\end{tabular}

Abbreviations: CDI, Cardiovascular Devices, Inc 3M; PB, Puritan Bennett; OP, Optex; B, bias; P, precision ( \pm SD of bias); r, correlation coefficient; $\mathrm{N}$, number of data points.

*These values are mean and SD of the absolute value of the differences. No bias information provided.

tStudy in 12 patients, 5 of 12 probes malfunctioned prior to end of study.

$\pm 6.06 \mathrm{mmHg}$ for $\mathrm{PCO}_{2}$, and $-5.89 \pm 13.19 \mathrm{mmHg}$ for $\mathrm{PO}_{2}$ (Table 2). The bias and precision values comparing the two blood gas machines were nearly identical to those comparing the blood gas machine to the optode sensor. ${ }^{23,24}$

In both the $\mathrm{CDI}^{22}$ and Optex ${ }^{23}$ studies probes were calibrated in vivo to laboratory determined arterial blood gases. No information is provided from either study as to the degree of correction required at each calibration (ie, what the drift was). Thrombus formation during use of an indwelling probe also remains a concern that has not been adequately answered to date. In the five patients studied with the Optex probe, the authors report no problems with arterial waveforms, and no evidence of thrombus. However, the number of patients remains small, and none of the studies in the literature specifically addresses thrombus formation as a study question. For example, no investigation included Doppler ultrasound evaluation of vessel patency following removal of the arterial cannula or probe. Sensors used in the CDI study were examined microscopically for thrombus, but no results are presented other than to state that no patients were removed from the study due to clot formation or sensor failure.

The literature contains two case reports of continuous blood gas monitoring. One case used a CDI probe during single-lung ventilation. ${ }^{25}$ The other related detection of venous air embolism during monitoring with a CDI probe. ${ }^{26}$ These reports may indicate the applicability of these monitoring devices to specific cases.

\section{Variations on a Theme: Current Models}

Based on the studies noted above, numerous companies are developing commercially available systems. Given the available technology, an ideal system would have the characteristics shown in Table 1. The issues of accuracy, bias, precision, drift, and performance of indwelling monitors over a 72 -hour period have not yet been addressed adequately, as no large in vivo studies have been reported on any of the systems. In fact, the total number of patients in whom probes have been placed and data collected and

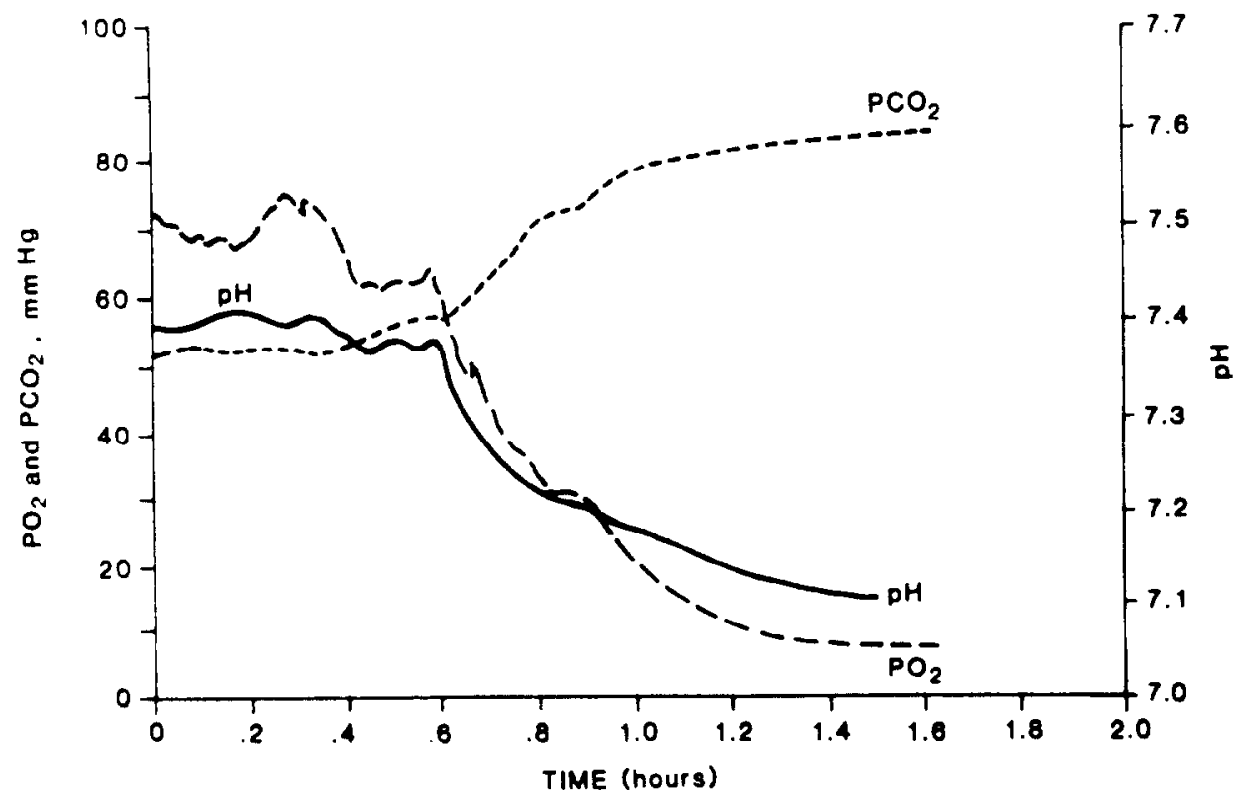

Fig 9. Pattern of acute changes in optode values observed in patients. The oxygen tension went down, the carbon dioxide went up, and the pH went down. Similar down-up-down patterns were observed when clots were induced at the probe tip in animal studies. (Reprinted with permission.20) 
A

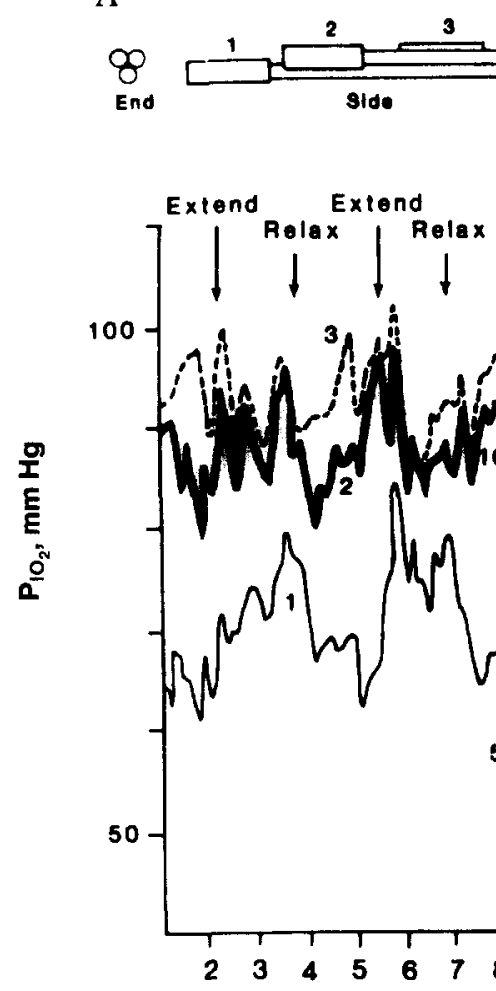

B

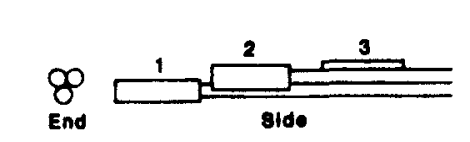

C

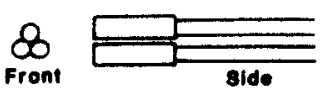
Rotale Rotate Bend Bend $180^{\circ}$ Relax $180^{\circ}-180^{\circ}$<smiles>[3H]I</smiles>

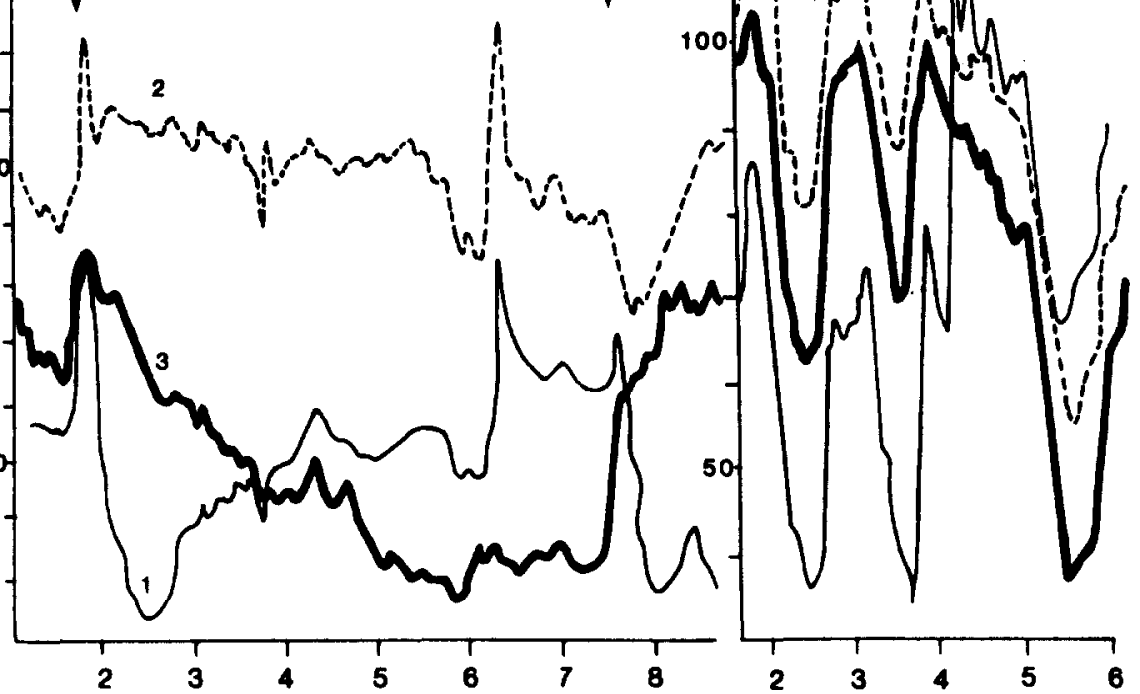

TIME EACH SEGMENT-6 minutes

Fig 10. Manipulations with probes consisting of three sensors of oxygen tension arranged in a staggered fashion (A and B) and side-by-side (sensors fiush at the probe tip) fashion (C). Each probe tip extended beyond the cannula tip. (A) Most distal sensor has the lowest oxygen tension and is most influenced by relaxation and hyperextension. The most distal sensor extended just beyond the cannula tip. (B) This shows that $180^{\circ}$ rotation raised the most distal oxygen tension above that of the most proximal sensor. A subsequent $180^{\circ}$ rotation reversed the situation. The most proximal sensor extended just beyond the cannula tip. (C) The same effect as in (B) can be obtained with $180^{\circ}$ rotation in three sensors flush at the probe tip. The probe tip extended slightly beyond the cannula tip. (Reprinted with permission. ${ }^{20}$ )

presented in peer-review journals appears to be less than 100. The CDI ex vivo system has been studied more extensively and appears to be accurate over 24 to 72 hours; even this system, however, has been used in only about 100 patients.

Finally, the most difficult question with this new technology, like many, may be cost/benefit analysis. Given the current health care monetary crisis and widespread discussion concerning apportionment of resources, a new technology needs to be cost-effective either in terms of improved mortality or cost savings. Unfortunately, often the capability to perform a test quickly becomes sole justification to do so, with no demonstrated improvement in mortality or morbidity. Muakkassa et $\mathrm{al}^{27}$ evaluated ABG use in a surgical ICU over a 1-year period and showed via multivariable analysis "that the presence of an A-line was the most powerful predictor of the number of $A B G s$ drawn per patient $(P<0.0001)$ regardless of all other measures of the patient's clinical status." The anticipated cost of each continuous blood gas monitor is $\$ 20,000$ with a per probe cost close to $\$ 400$. The indwelling probe will not obviate blood gas determinations that will have to be done at least every 24 hours for calibration. The total cost per 24 hour period, assuming one probe is in use and valuable for 72 hours, will approximate the cost of four to five arterial blood gas determinations. If a probe is used for only 24 hours, the cost bccomes closer to that of seven to eight arterial blood gases. Finally, any cost-benefit analysis must take into consideration complication rate, such as arterial thrombus, increased nursing time to prevent or treat complications, and time required to maintain the equipment.

In selected patients, the probes may prove to be costeffective, although this evaluation cannot be made with any certainty until large-scale studies have been completed and the true costs are known. A clear concern is that the number of arterial blood gas determinations may not decrease despite the indwelling continuous probe.

Puritan Bennett FOxS Sensor. (FOxS Division, Carlsbad, $C A)$. This sensor uses fluorescence to determine oxygen tension, and absorbance technology to determine $\mathrm{pH}$ and carbon dioxide tension. The analyzing dyes are impregnated in the surface cladding of the probe, and back from the tip (Fig 5), potentially correcting wall effect problems. The FOxS is currently available. Larson et $\mathrm{al}^{28}$ evaluated nine probes used in critically ill patients and found a mean absolute drift of 3 to $6 \mathrm{mmHg}$ for $\mathrm{PO}_{2}, 4$ to $7 \mathrm{mmHg}$ for 
$\mathrm{PCO}_{2}$, and 0.03 to 0.05 for $\mathrm{pH}$ measurement. No values are reported for bias or precision.

CDI-3M Healthcare System 2000. As noted above, Shapiro et al presented their experience with this probe in 117 patients with good results. More experience is available with a conceptually similar system, the (CDI, Irvine, CA), Gas-STAT a flow-through fluorometric system used to monitor blood gas values in the cardiopulmonary bypass circuit. Bashein et $\mathrm{al}^{29}$ and Pino et $\mathrm{al}^{30}$ reported results both in vitro and in vivo with the Gas-STAT system and concluded that whereas "the monitor is shown to be a valuable indicator of trends ... its accuracy in the flowthrough environment does not match its calibration performance and is insufficient to supplant traditional analyses of discrete samples for absolute measurements." Peruzzi et al ${ }^{31}$ evaluated the CDI 2000 system in an unspecified number of patients and reported bias and precision values as follows: $\mathrm{pH} 0.004 \pm 0.025, \mathrm{PO}_{2} 0.9 \pm 2.3 \mathrm{mmHg}$, and $\mathrm{PCO}_{2} 2.8 \pm 9.9 \mathrm{mmHg}$.

Optex Biomedical Biosentry System. Rather than using glass fibers for light transmission, this sensor uses plastic fibers that may be bent $180^{\circ}$ without breaking. The indicator dye chamber is located along the path of the light at the side of the probe (Fig 5C), again reducing thrombogenicity and decreasing wall effect problems. Zimmerman and Dellinger have presented their experience with this probe in five patients as noted above, ${ }^{2.3}$ and in other patients presented in non-peer-review literature. ${ }^{32}$

Pfizer Paratrend 7 (Pfizer Biomedical Sensors, Malvern, $P A)$. This system uses a Clark polarographic electrode for determination of $\mathrm{PaO}_{2}$ and absorbance technology for determination of $\mathrm{pH}$ and $\mathrm{PaCO}_{2}$. Early studies using miniaturized Clark electrodes, as noted above, experienced problems related to thrombus formation and interference with transduced pressures, presumably secondary to size of the electrode. No data exist in the literature to date on the in vivo performance of this system.

\section{SUMMARY}

The benefit of a continuous intra-arterial blood gas monitor is apparent to any physician who has ever waited for the return of blood gas values at the bedside of a critically ill patient. The potential for rapid detection and prompt treatment of blood gas changes, coupled with immediate evaluation of the effectiveness of a therapeutic intervention, appears great. Whereas these systems may offer a tremendous advance in the physician's ability to track and treat alterations in cardiopulmonary physiology, no large-scale in vivo human trials have yet been reported. The only data available on accuracy, bias, precision, drift, and complication rate are from relatively small trials. Hopefully, when large-scale trials are completed, these systems will live up to their tremendous potential.

\section{REFERENCES}

1. Clark LC Jr: Monitor and control of tissue oxygen tensions. Trans Am Society Artific Intern Organs 2:41-48, 1956

2. Severinghaus JW, Bradley AF: Electrodes for blood $\mathrm{PO}_{2}$ and $\mathrm{PCO}_{2}$ determination. J Applied Physiology 13:515-520, 1958

3. Kollmeyer KR, Tsang RC: Complications of umbilical oxygen electrodes. J Pediatr 84:894-897, 1974

4. Bratanow N, Polk K, Bland R, et al: Continuous polarographic monitoring of intra-arterial oxygen in the perioperative period. Crit Care Med 13:859-860, 1985

5. Katayama M, Murray GC, Uchida $T$, et al: Intra-arterial continuous monitoring by an ultra-fine microelectrode. Crit Care Med 15:357, 1987

6. Tremper KK, Barker SJ: Fundamental principles of monitoring instrumentation, in Miller R (ed): Anesthesia, ed 3. New York, NY, Churchill-Livingston, 1990, p 986

7. Gehrich JL, Lubbers DW, Opitz N, et al: Optical fluorescence and its application to an intravascular blood gas monitoring system. IEEE Transactions on Biomedical Engineering. BME-33:117-132, 1986

8. Tremper KK, Barker SJ: Fundamental principles of monitoring instrumentation, in Miller R (ed): Anesthesia (ed 3). ChurchillLivingston, New York, NY, 1990, p 985

9. Kautsky H: Quenching of luminescence by oxygen. Trans Faraday Soc 35:216-219, 1939

10. Opitz N, Lubbers DW: Theory and development of fluorescent based photochemical oxygen sensors: Oxygen optodes. Intern Anesth Clin 25:177-197, 1987

11. Peterson JL, Vurek GG: Fiberoptic sensors for biomedical applications. Science 224:123-127, 1984

12. Lippitsch ME, Pusterhofer J, Lciner MJ, Wolfbeis OS: Fiberoptic oxygen sensor with fluorescence decay time as information carrier. Analytica Chimica Attica 205:1-6, 1988
13. Saari LA, Seitz WR: pH sensor based upon immobilized fluoresceinamine. Annal Chem 54:821-825, 1982

14. Peterson JL, Goldstein SR, Fitzgerald RV, Buckhold DK: Fiberoptic $\mathrm{pH}$ probe for physiological use. Anal Chem 52:864-869, 1980

15. Gottlieb A, Divers S, Hui HK: In vivo applications of fiberoptic chemical sensors, in Wise DL, Wingard LB, Jr (eds): Biosensors with Fiberoptics. Clifton, NJ, Humana, 1991

16. Shapiro BA, Harrison RA, Cane RD, et al: Quality assurance in blood gas analysis, in Shipiro BA (ed): Clinical Application of Blood Gases (ed 4). Chicago, IL, Year Book, 1989, pp 274-282

17. Thorson SH, Marini JJ, Pierson DJ, et al: Variability of arterial blood gas values in stable patients in the ICU. Chest 84:14-18, 1983

18. Bland JM, Altman DG: Statistical methods for assessing agreement between two methods of clinical measurement. Lancet 1:307-310, 1986

19. Shapiro BA, Cane RD, Chomka CM, et al: Preliminary evaluation of an intra-artrial blood gas system in dogs and humans. Crit Care Med 17:455-460, 1989

20. Mahutte CK, Sassoon SH, Muro JR, et al: Progress in the development of a fluorescent intravascular blood gas system in man. I Clin Monit 6:147-157, 1990

21. Barker SJ, Hyatt J: Continuous measurement of intraarterial $\mathrm{pHa}, \mathrm{PaCO}_{2}$, and $\mathrm{PaO}_{2}$ in the operating room. Anesth Analg 73:43-48, 1991

22. Shapiro BA, Mahutte CK, Cane RD, Gilmour IJ: Clinical performance of a blood gas monitor: A prospective, multicenter trial. Crit Care Med 21:487-494, 1993

23. Zimmerman JL, Dellinger RP: Initial evaluation of a new intra-arterial blood gas system in humans. Crit Care Med 21:495500,1993

24. Zimmerman JL, Dellinger RP: Evaluation of a continuous 
intra-arterial blood gas system in critically ill patients. Chest 102:105S, 1992

25. Greenblott GB, Tremper KK, Barker SJ, et al: Continuous blood gas monitoring with an intraarterial optode during one-lung anesthesia. J Cardiothorac Vasc Anesth 5:365-367, 1991

26. Greenblott GB, Barker SJ, Tremper KK, et al: Detection of venous air embolism by continuous intraarterial oxygen monitoring. J Clin Monit 6:53-56, 1990

27. Muakkassa FF, Rutledge R, Fakhry SM, et al: ABGs and arterial lines: The relationship to unnecessarily drawn arterial blood gas samples. J Trauma 30:1087-1095, 1990

28. Larson CP, Siever A, Vender J: Evaluation of drift in an intra-arterial blood gas sensor. Anesthesiology 77:A538, 1992
29. Bashein G, Pino JA, Nessly ML, et al: Clinical assessment of a flow-through fluorometric blood gas monitor. J Clin Monit 4:195-203, 1988

30. Pino JA, Bashein G, Kenny MA: In vitro assessment of a flow-through fluorometric blood gas monitor. J Clin Monit 4:186194,1988

31. Peruzzi WT, Shapiro BA, Cane RD: Evaluation of a rapid, on-demand, ex-vivo, arterial blood gas monitoring system. Chest 102:105S, 1992

32. Dellinger RP, Zimmerman JL: Continuous intra-arterial blood gas monitoring, in Vincent JL (ed): 1992 Yearbook of Intensive Care and Emergency Medicine. Berlin, Germany, Springer-Verlag, 1992, pp 401-410 